\title{
Stability Analysis of Anonlinear Autoregressive Model of First Order
}

Abdulghafoor Gasim Salim

drabdul_salim@uomosul.edu.iq
Abeer Abdulkhaliq Ahmed

abeerdabagh1@gmail.com

College of Computer Sciences and Mathematics

University of Mosul, Mosul, Iraq

Received on: 02/01/2013

Accepted on 03/04/2013

\section{ABSTRACT}

In this paper, we study the moment of a non linear - autoregressive polynomial model of first order, also we analysis the stability of this model and find the singular point as well as the limit cycle by using the linear approximation technique.

Keywords: the singular point, the limit cycle, the linear approximation technique

$$
\begin{aligned}
& \text { تحليل إستقرارية أنموذج أنحدار ذاتي غير خطي من المرتبة الأولى } \\
& \text { عبير عبل الخالق احدد } \\
& \text { عبد الغفور جاسم سالم } \\
& \text { قسم الرياضيات } \\
& \text { كلية علوم الحاسوب والرياضيات } \\
& \text { جامعة الموصل، الموصل، العراق }
\end{aligned}
$$

\section{الملخص}

تم في هذا البحث دراسـة العزوم لأنموذج الأنحدار الذاتي غير الخطي متعدد الحدود من المرتبة الأولى

وكذلك تم تحليل إستقرارية هذا الأنموذج وإيجاد النقطة الثابتة ودورة النهاية باستخدام تقنية التقريب الخطية.

الكلمات المفتاحية: النقطة المنفردة، دورة النهاية، طريقة التقريب بالخطية المحلية.

1 - 1 - 1 - 1

السلاسل الزمنية هي سجل للقيم لأي كمية متقلبة تقاس بنقاط مختلفة من الزمن. على سبيل المثال قياس

درجات الحرارة اليومية ، قياس الفولطية للدائرة الكهبائية لكل ثأنية واحدة ، قياس مؤشرات الأسعار لكل شهر ...

عادة تبنى السلاسل الزمنية على ميزات أساسية متمثلة بالإستقرارية (stability) والخطية (linearity) والطبيعية (normality) وكيفية معالجة السلاسل الزمنية غير المراوحة لبناء نماذج رياضية ملائمة لها ، سيتركز بحثا على دراسة إستقرارية احد النماذج غير الخطية (المتعدد الحدود من المرتبة الأولى ).وبما أن هذا الأنموذج لهاء

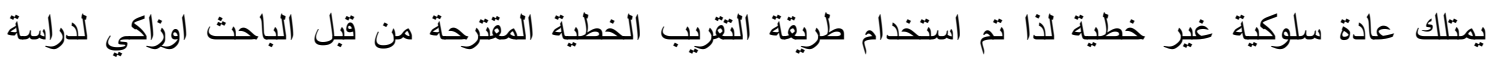

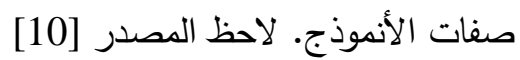


2- تعاريف أساسية

1-2](time series) المتسلسلة الزمنية)

هي مجموعة من المشاهدات التي تتولد على التوالي خلال الزمن ، وتتميز أي سلسلة زمنية بأن بيأناتها

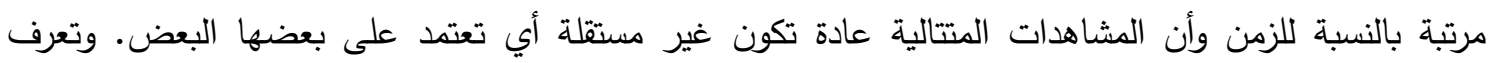
العملية التصادفية بأنها عائلة من المتغيرات العشوائية

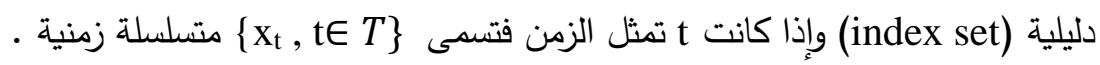

2-2 نماذج الأنحدار الذاتي والأوساط المتحركة]

\section{Autoregressive moving average model $\operatorname{ARMA}(p, q)$}

لتكن $=\mathrm{f}\left(X_{t-1}, \ldots, X_{t-p}, Z_{t-1}, \ldots, Z_{t-q}\right)+Z_{t} \quad \ldots \quad$ (1) $X_{t}$

إذ أن z(t) هي الضوضاء الأبيض (الضجيج الأبيض) وتتبع التوزيع الطبيعي بمعدل صفر وتباين f(.),

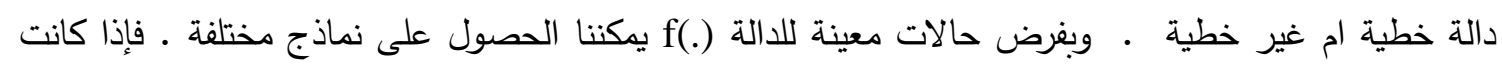

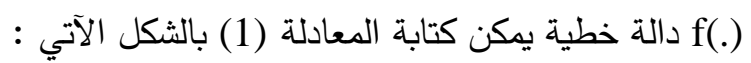

(2) $X_{t}=\mathrm{a}_{0}+\sum_{i=1}^{p} a_{i} X_{t-i}+\sum_{i=1}^{q} b_{i} Z_{t-i}+Z_{t}$

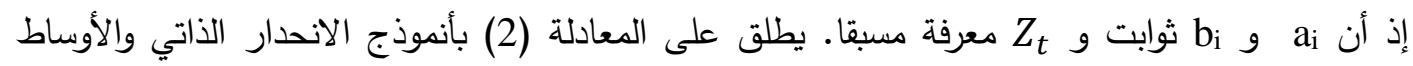

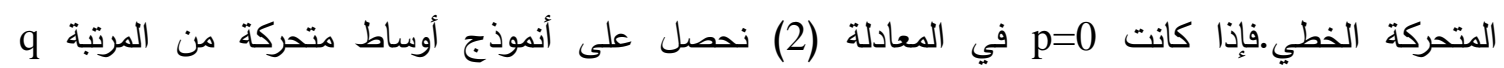

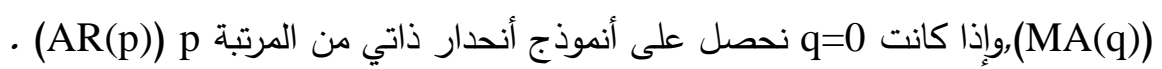

3- أنموذج متعدد الحدود للانحدار الذاتي والأوساط المتحركة:[8]

لتكن $x_{(t)}=f\left(x_{(t-1)}, x_{(t-p)}, z_{(t-1)}, \ldots, z_{(t-p)}\right)+z_{t}$

$x_{(t)}=p\left(x_{(t-1)}, \ldots, x_{(t-p)}, z_{(t-1)}, \ldots, z_{(t-q)}\right)+z_{(t)}$

إذ إن (.) هي متعددة حدود للمتغيرات

بانموذج متعدد الحدود للانحدار الذاتي والأوساط المتحركة.

المعادلة (4)

4-2 أنموذج الانحدار الذاتي من المرتبة الأولى غير الخطي متعدد الحدود(الأنموذج المقترح):

لتكن المتسلسلة الزمنية $\}$ Xيث المرتبة الاولى بالثكل الأتي:

$+z_{t} \quad, k=2,3, \ldots$

... (5) $X_{\mathrm{t}}=\left(a X_{t-1}\right)^{\mathrm{k}-1} \mathrm{X}_{\mathrm{t}-1}$

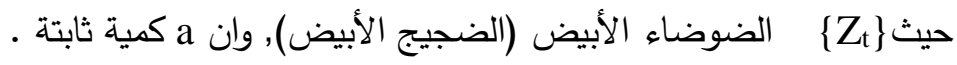

2-5 أنموذج الأنحدار الأتي الأسي :

عرف Oda and Ozaki عام 1977 أنموذج الانحدار الذاتي الاسي من المرتبة pبالثكل الأتي $\mathrm{X}_{\mathrm{t}}=\sum_{i=1}^{p}\left(\alpha_{\mathrm{i}}+\pi_{\mathrm{i}} e^{-x_{i-1}^{2}}\right) x_{t-i}+Z_{t}, \mathrm{i}=1,2,3, \ldots, \mathrm{p}$ 
حيث $\alpha_{i}, \pi_{i}$ كميات ثابتة (معلمات الأنموذج).

وقد اثبت العالمان ( Oda and Ozaki ) إستقرارية دورة النهاية للأنموذج (5) يمكن ايجادها من خلال

المبرهنة الآتية :

: 6 6برهنة

لتكن المتسلسلة الزمنية $\left\{X_{t}\right.$ متمثلة بأنموذج الأنحدار الذاتي الاسي من المرتبة الأولى :

$\mathrm{X}_{\mathrm{t}}=\left(\alpha_{1}+\pi_{1} e^{-x_{t-1}^{2}}\right) x_{t-1}+Z_{t}$

دورة النهاية بدورة, للأنموذج تكون مستقرة مداريا (orbitally stable X

$<1\left|\prod_{j=1}^{q}\left[\alpha_{j}+\pi_{j}\left(1-2 x_{t+q-j}^{2}\right) e^{-x_{t+q-j}^{2}}\right]\right|$

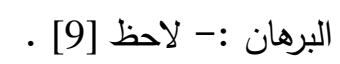

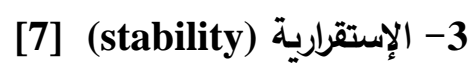

تصادفنا في العديد من المسائل الفيزيائية والهنسية عمليات عثوائية يمكن وصفها على نحو غير ثابت

وغالبا ما تكون هذه العمليات في حالة توازن إحصائي)(Statistical equilibrium)

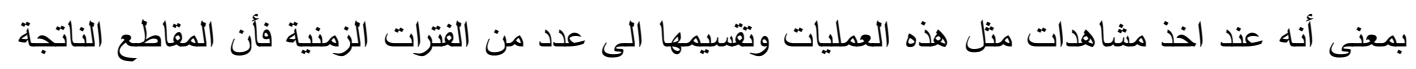
تمتلك خصائص احتمالية وإحصائية متثابهة.

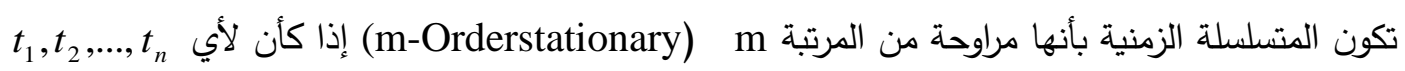
ولأي ثابت k فأن جميع العزوم من الارجة $E\left[\left\{X_{t_{1}}\right\}^{m_{1}},\left\{X_{t_{2}}\right\}^{m_{2}}, \ldots,\left\{X_{t_{n}}\right\}^{m_{n}}\right]=E\left[\left\{X_{t_{1+k}}\right\}^{m_{1}},\left\{X_{t_{2+k}}\right\}^{m_{2}}, \ldots,\left\{X_{t_{n+k}}\right\}^{m_{n}}\right]$

الزمنية المختارة الأس الأولى (First Order Stationary) إذا كأنت:

كما يقال اللسلسلة الزمنية حققت الشروط الآتية: (كال

1) $E\left(X_{t}\right)=\mu$

$t$ كمية ثابتة لاتعتمد على 4

2) $\operatorname{Var}\left(X_{t}\right)=\sigma_{X}^{2}$

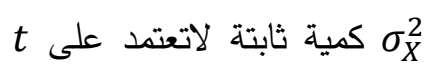

3) $\operatorname{Cov}\left[X_{t 1}, X_{t 2}\right]=\gamma_{(t 1, t 2)}$ دالة بدلالة

1-3 النقطة المنفردة (singular point):

النقطة الدنفردة تعرف بأنها تلك النقطة التي تحقق الثرط الآتي: إن أي مسار للنموذج يبدأ من نقطة

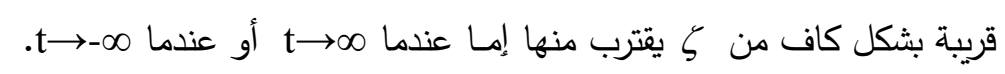

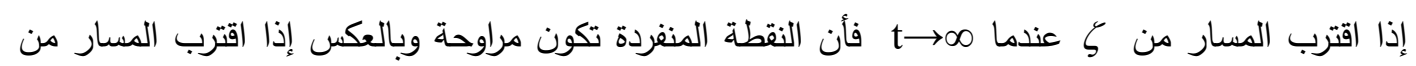

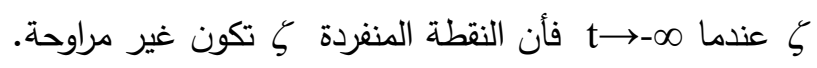


[12] - 2-3 دورة النهاية (limit cycle)

q دورة النهاية للنموذج تعرف بأنها المسار المغلق والمعزول عدد صحيح موجب. المقصود بأنَّ المسار مغلق هو أنه إذا كأنت القيم الابتدائية (x) تنتمي إلى دورة النهاية فأن

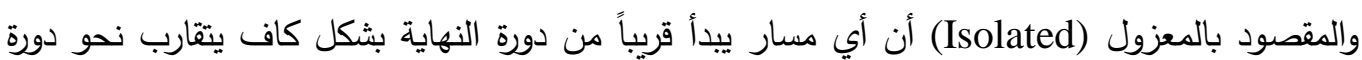

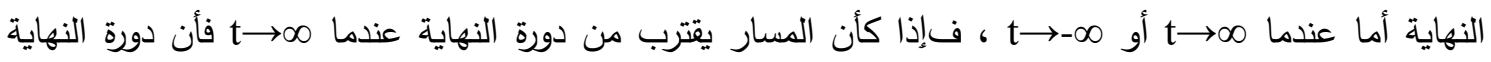
تكون مراوحة وبالعكس إذا كأن المسار يقترب من دورة النهاية عندما ما مأه فأن دورة النهاية تكون غير مراوحة.

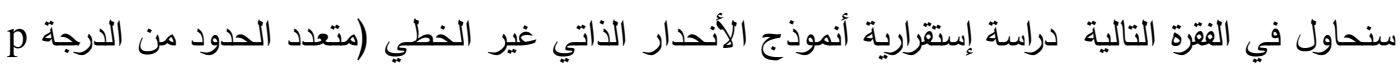

والمرتبة الأولى)

3-3 الصفات الأحصائية للأنموذج المقترح :

ليكن لدينا أنموذج أنحدار ذاتي غير خطي (متعدد الحدود) من الدرجة k و المرتبة الاولى: $\mathrm{X}_{\mathrm{t}}=(\mathrm{a} \mathrm{X} \mathrm{t}-1)^{\mathrm{k}-1} \mathrm{X}_{\mathrm{t}-1}+\mathrm{Z}_{\mathrm{t}} \quad \mathrm{k}=2,3,4, \ldots$.

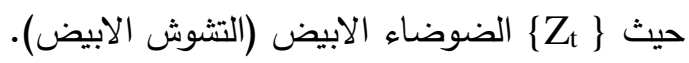

سنحاول إيجاد الصفات الإحصائية للأنموذج (8)بتحويله الى انموذج اوساط متحركة باستخدام طريقة التقريب المتعاقب وبفرض أن k=2,3 ثم نعمم النتائج ·

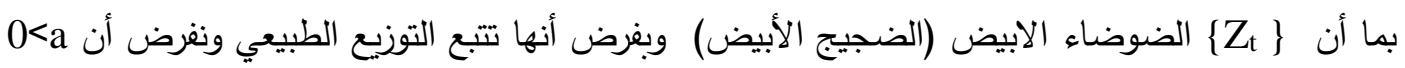

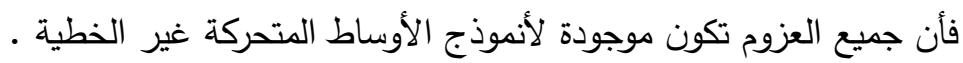
أولا: نضع k=2 في الأنموذج (8) نحصل على أنموذج متعدد حدود من الدرجة الثانية والمرتبة الاولى: لانئ $X_{t}=\left(a X_{t-1}\right)^{2}+Z_{t}$ وبفرض أن Xo=0

$\mathrm{X}_{1}=\mathrm{Z}_{1}$

$\mathrm{X}_{2}=\mathrm{a} Z_{1}^{2}+Z_{2}$

$X_{t}=Z_{t}+\sum_{j=0}^{t-2} a^{2^{j}-1} Z_{t-j-1}^{2^{j}}+\sum_{u=0}^{\infty} \sum_{v=0}^{\infty} g_{u v} Z_{t-u} Z_{t-v}$

$+\sum_{u=0}^{\infty} \sum_{v=0}^{\infty} \sum_{w=0}^{\infty} g_{u v w} Z_{t-u} Z_{t-v} Z_{t-w}+\cdots$

حيث (.) وتمثل الثابت a بصيغ معينة كناتج تبسيط مفكوك المعادلة أعلاه .المعادلة (9) تمثل أنموذج التمثيل غير

الخطي العام(General non-linear representation)

سنحاول في هذه الفقرة التالية إيجاد الصفات الإحصائية للأنموذج (9):

أولا: القيمة المتوقعة: بأخذ القيمة المتوقعة للمعادلة (9) نحصل على لهى 


$$
\begin{aligned}
& E\left(X_{t}\right)=E\left(Z_{t}\right)+E\left(\sum_{j=0}^{t-2} a^{2^{j}-1} Z_{t-j-1}^{2^{j}}\right) \\
& +E\left(\sum_{u=0}^{\infty} \sum_{v=0}^{\infty} g_{u v} Z_{t-u} Z_{t-v}+\sum_{u=0}^{\infty} \sum_{v=0}^{\infty} \sum_{w=0}^{\infty} g_{u v w} Z_{t-u} Z_{t-v} Z_{t-w}\right. \\
& +\cdots) \\
& \text { ضجيج ابيض } \\
& \therefore E\left(X_{t}\right)=0+E\left(\sum_{j=0}^{t-2} a^{2^{j}-1} Z_{t-j-1}^{2^{j}}\right)+0 \\
& )=\sum_{j=0}^{t-2} a^{2^{j}-1} E\left(Z_{t-j-1}^{2^{j}}\right) E\left(\sum_{j=0}^{t-2} a^{2^{j}-1} Z_{t-j-1}^{2^{j}}\right. \\
& E\left(Z^{2^{j}}\right)=\int_{-\infty}^{\infty} Z^{2^{j}} f(z) d z=2 \int_{0}^{\infty} Z^{2^{j}} f(z) d z \because \\
& )=2 \int_{0}^{\infty} Z^{2^{j}} \frac{1}{\sqrt{2 \pi}} e^{-\frac{1}{2} z^{2}} d z
\end{aligned}
$$

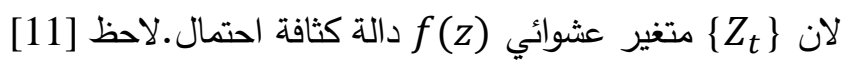

$$
\begin{aligned}
& \left(\mathrm{Z} \sim N\left(0, \sigma_{z}^{2}\right)\right) \Rightarrow E\left(Z^{2^{j}}\right.
\end{aligned}
$$

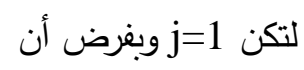

$$
\begin{aligned}
& \Rightarrow z=\sqrt{2 u} \Rightarrow d z=\frac{d u 1}{\sqrt{2 u} 2} z^{2}=u \\
& )=2 \int_{0}^{\infty} 2 u \frac{1}{\sqrt{2 \pi}} e^{-u} \frac{d u}{\sqrt{2 u}} \cdot \therefore\left(Z^{2}\right. \\
& =\frac{2}{\sqrt{\pi}} \int_{0}^{\infty} u^{\frac{1}{2}} e^{-u} d u \\
& =\frac{2}{\sqrt{\pi}} \frac{1}{2} \Gamma \frac{1}{2}=1 \\
& )=2 \int_{0}^{\infty} Z^{2^{t-2}} \frac{1}{\sqrt{2 \pi}} e^{-\frac{1}{2} z^{2}} d z E\left(Z^{2^{t-2}}\right. \\
& )=\left(\frac{2^{t-\frac{3}{2}}}{\sqrt{2 \pi}}\right) \Gamma\left(t-\frac{5}{2}\right) E\left(Z^{2^{t-2}}\right. \\
& \mathrm{t}=3 \Rightarrow E\left(Z^{2^{3-2}}\right)=\left(\frac{2^{3-\frac{3}{2}}}{\sqrt{2 \pi}}\right) \Gamma\left(3-\frac{5}{2}\right)=\left(\frac{2 \sqrt{2}}{\sqrt{2} \sqrt{\pi}}\right) \Gamma \frac{1}{2}=2 \text { لتكن } \\
& )=\mathrm{g}(\mathrm{t}) \Rightarrow \sum_{j=0}^{t-2} a^{2^{j}-1} E\left(Z_{t-j-1}^{2^{j}}\right. \\
& \mathrm{g}(\mathrm{t}) \quad \Longrightarrow E\left(X_{t}\right)= \\
& \operatorname{Var}\left(X_{t}\right)=E\left(X_{t}^{2}\right)-E\left(X_{t}\right)^{2} \because \\
& X_{t}^{2}=Z_{t}^{2}+\sum_{j=0}^{t-2} a^{4^{j+1}-2} Z_{t-j-1}^{4^{j}}+\sum_{u=0}^{\infty} \sum_{v=0}^{\infty} g_{u v} Z_{t-u} Z_{t-v} \\
& +\sum_{u=0}^{\infty} \sum_{v=0}^{\infty} \sum_{w=0}^{\infty} g_{u v w} Z_{t-u} Z_{t-v} Z_{t-w}+\cdots
\end{aligned}
$$

وبأخذ القيمة المتوقعة للمعادلة (11) نحصل على 


$$
\begin{aligned}
& E\left(X_{t}^{2}\right)=E\left(Z_{t}^{2}\right)+E\left(\sum_{j=0}^{t-2} a^{4^{j+1}-2} Z_{t-j-1}^{4^{j}}\right)+E\left(\sum_{u=0}^{\infty} \sum_{v=0}^{\infty} g_{u v} Z_{t-u} Z_{t-v}\right) \\
& +E\left(\sum_{u=0}^{\infty} \sum_{v=0}^{\infty} \sum_{w=0}^{\infty} g_{u v w} Z_{t-u} Z_{t-v} Z_{t-w}\right)+\cdots \\
& \because E\left(\sum_{j=0}^{t-2} a^{4^{j+1}-2} Z_{t-j-1}^{4^{j}}\right)=\sum_{j=0}^{t-2} a^{4^{j+1}-2} E\left(Z_{t-j-1}^{4^{j}}\right) \\
& \text { وباستخدام نفس العلاقات والفرضيات السابقة نجد أن } \\
& E\left(X_{t}^{2}\right)=h(t) \\
& \text { حيث (th) دالة تعتمد على t .وهذا يعني ان العملية غير مراوحة. }
\end{aligned}
$$

ثالثا : التغاير :

$$
\begin{aligned}
& \because \operatorname{Cov}\left(X_{t}, X_{t \pm k}\right)=E\left(X_{t} \cdot X_{t \pm k}\right)-E\left(X_{t}\right) \cdot E\left(X_{t \pm k}\right) \\
& X_{t \pm k}=\sum_{j=0}^{t-2} a^{p^{j}-r} Z_{t \pm K-j-1}^{p^{j}}+\sum_{u=0}^{\infty} \sum_{v=0}^{\infty} g_{u v} Z_{t \pm K-u} Z_{t \pm K-v} \\
& \quad+\sum_{u=0}^{\infty} \sum_{v=0}^{\infty} \sum_{w=0}^{\infty} g_{u v w} Z_{t \pm K-u} Z_{t \pm K-v} Z_{t \pm K-w}
\end{aligned}
$$

حيث p,r p ميات ثابتة.

$$
\begin{aligned}
X_{t} \cdot X_{t \pm k}=\sum_{j=0}^{t-2} a^{q^{j}-b} Z_{t \pm K-j-1}^{q^{j}}+\sum_{u=0}^{\infty} \sum_{v=0}^{\infty} g_{u v} Z_{t \pm K-u} Z_{t \pm K-v} \\
+\sum_{u=0}^{\infty} \sum_{v=0}^{\infty} \sum_{w=0}^{\infty} g_{u v w} Z_{t \pm K-u} Z_{t \pm K-v} Z_{t \pm K-w}
\end{aligned}
$$

حيث q,b وميات ثابتة.

$\mathrm{E}\left(X_{t} \cdot X_{t \pm k}\right)=l(t)$

وبأخذ القيمة المتوقعة للمعادلة الأخيرة نحصل على

أي أن التغاير يعتمد على t وأن t تمثل الزمن • نستتتج من الفقرة السابقة أن عزوم الأنموذج الذاتي غير الخطي المتعدد الحدود من المرتبة الاولى جميعها تعتمد على الزمن أي أن الأنموذج غير مراوح. ثانيا: سوف نحاول إيجاد العزوم للأنموذج (8) عندما k=3 (انموذج متعدد الحدود من الدرجة الثالثة $\mathrm{X}_{\mathrm{t}}=(\mathrm{a} \mathrm{X} \mathrm{t}-1)^{3}+\mathrm{Z}_{\mathrm{t}}$

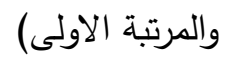
وباستخدام نفس الفرضية السابقة نحصل على 


$$
\begin{aligned}
X_{t}=Z_{t}+\sum_{j=0}^{t-2} a^{3^{j}-1} Z_{t-j-1}^{3^{j}}+\sum_{u=0}^{\infty} \sum_{v=0}^{\infty} g_{u v} Z_{t-u} Z_{t-v} \\
+\sum_{u=0}^{\infty} \sum_{v=0}^{\infty} \sum_{w=0}^{\infty} g_{u v w} Z_{t-u} Z_{t-v} Z_{t-w}+\cdots
\end{aligned}
$$

القيمة المتوقعة:

$$
\begin{array}{r}
E\left(X_{t}\right)=E\left(Z_{t}+\sum_{j=0}^{t-2} a^{3^{j}-1} Z_{t-j-1}^{3^{j}}+\sum_{u=0}^{\infty} \sum_{v=0}^{\infty} g_{u v} Z_{t-u} Z_{t-v}\right. \\
\left.+\sum_{u=0}^{\infty} \sum_{v=0}^{\infty} \sum_{w=0}^{\infty} g_{u v w} Z_{t-u} Z_{t-v} Z_{t-w}+\cdots\right)
\end{array}
$$

$$
\begin{gathered}
E\left(X_{t}\right)=E\left(Z_{t}\right)+E\left(\sum_{j=0}^{t-2} a^{3^{j}-1} Z_{t-j-1}^{3^{j}}\right)+E\left(\sum_{u=0}^{\infty} \sum_{v=0}^{\infty} g_{u v} Z_{t-u} Z_{t-v}\right) \\
+E\left(\sum_{u=0}^{\infty} \sum_{v=0}^{\infty} \sum_{w=0}^{\infty} g_{u v w} Z_{t-u} Z_{t-v} Z_{t-w}\right)+\cdots
\end{gathered}
$$

$E\left(Z_{t}\right)=0$

$E\left(Z^{3^{j}}\right)=2 \int_{0}^{\infty} Z^{3^{j}} \frac{1}{\sqrt{2 \pi}} e^{-\frac{1}{2} z^{2}} d z$

$\therefore E\left(X_{t}\right)=k(t)$

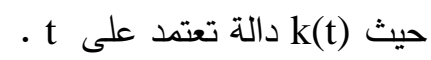

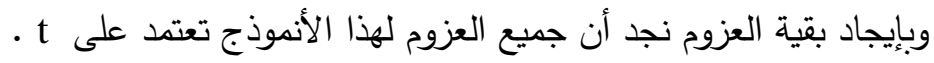

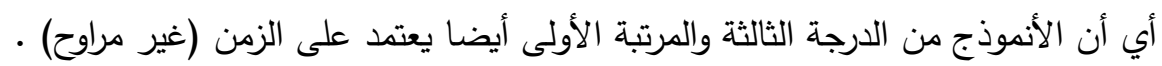
$\mathrm{X}_{\mathrm{t}}=\left(\mathrm{a} \mathrm{X}_{\mathrm{t}-1}\right)^{\mathrm{k}}{ }_{1}+\mathrm{Z}_{\mathrm{t}}$

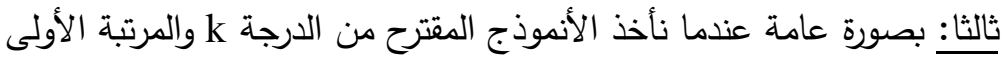
وباستخدام نفس الفرضيات السابقة للنماذج بدرجات دنيا عندما k=2,3) نحصل على

$$
\begin{array}{r}
X_{t}=Z_{t}+\sum_{j=0}^{t-2} a^{(k+1)^{j}-1} Z_{t-j-1}^{(k+1)^{j}}+\sum_{u=0}^{\infty} \sum_{v=0}^{\infty} g_{u v} Z_{t-u} Z_{t-v} \\
+\sum_{u=0}^{\infty} \sum_{v=0}^{\infty} \sum_{w=0}^{\infty} g_{u v w} Z_{t-u} Z_{t-v} Z_{t-w}+\cdots
\end{array}
$$

المعادلة (18) تمثل أنموذج أوساط متحركة غير منتهي وغير خطي, وعند إيجاد العزوم للأنموذج السابق نحصل على نفس النتيجة. k نستنتج من ذلك أن الصفات الإحصائية لأنموذج الانحدار الذاتي غير الخطي(متعدد الحدود من الدرجة الإهن والمرتبة الأولى) يعتمد على الزمن, أي أن الأنموذج غير مراوح. 
3-4 طريقة التقربب: [10]

سنحاول في هذه الفقرة دراسة إستقرارية الأنموذج المقترح باستخدام التقريب بالمحلية غير الخطية: تتلخص الطريقة بالمراحل الآتية:

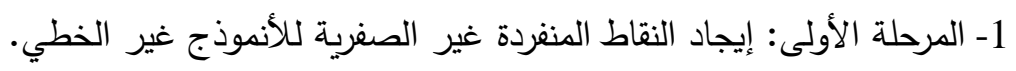
2- المرحلة الثانية: اختبار إستقرارية تلك النقطة باستخدام تقنية التقريب الخطية. 3- المرحلة الثالثة:- اختبار إستقرارية دورة النهاية ان وجدت.

$\mathrm{X}_{\mathrm{t}}=(\mathrm{a} \mathrm{X} \mathrm{t}-1)^{\mathrm{k}}+\mathrm{Z}_{\mathrm{t}}$

ليكن لدينا الأنموذج المعرف بالمعادلة (7) سنحاول دراسة إستقرارية الأنموذج عندما k=2,3 ثم نعم النتائج:

1-4-3 إيجاد النقطة المنفردة:

ع

أي أن

$\mathrm{X}_{\mathrm{t}}=\left(a X_{t-1}\right)^{2}+\mathrm{Z}_{\mathrm{t}}$

باستخدام تعريف النقطة المنفردة وإهمال تأثير $\}$ Z

$\Rightarrow \xi-a \xi^{2}=0 \Rightarrow \xi(1-a \xi)=0 \xi=a \xi^{2}$

$\xi=\frac{1}{\mathrm{a}} ; \xi=0$

أي أن هنالك نقطتان هما النقطة الثابتة الصفرية والنقطة الثابتة

3-3-3 إستقرارية النقطة المنفردة:

لأختبار استقرارية النقطة المنفردة , ليكن Xt تقترب من النقطة المنفردة ويمكن تمثيلها بالعلاقة

و $X_{t-1}=\xi+\xi_{t-1}=\xi+\xi_{t}$

$\xi+\xi_{t}=a\left(\xi+\xi_{t-1}\right)^{2}$

وبالتعويض بالأنموذج (19) نحصل على

$\xi+\xi_{t}=a\left(\xi^{2}+2 \xi \xi_{t-1}+\xi_{t-1}^{2}\right)$

$\xi_{t}=2 a \xi \xi_{t-1}$

$\because \xi=\frac{1}{\mathrm{a}}$

n ب أن

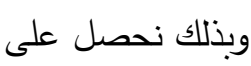

المعادلة (20) تمثل أنموذج انحدار ذاتي خطي من المرتبة الأولى وأن( جذر المعادلة يساوي 2 ) أي أنه

$$
\ldots(20) \xi_{t}=2 \xi_{t-1}
$$

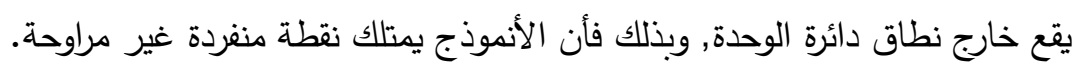

3-3-3 شرط إستقرارية دورة النهاية :

سنحاول في هذه الفقرة إيجاد شروط إستقرارية دورة النهاية (أن وجدت) للأنموذج المقترح والذي صيغته : $\mathrm{X}_{\mathrm{t}}=\left(\mathrm{a} \mathrm{X}_{\mathrm{t}-1}\right)^{\mathrm{k}-1} \mathrm{X}_{\mathrm{t}-1}+\mathrm{Z}_{\mathrm{t}}$

وبالاعتماد على المبرهنة (3-4) من خلال القضية الآتية: 
1-3-4-3

$\mathrm{X}_{\mathrm{t}}=\left(\mathrm{a} \mathrm{X}_{\mathrm{t}-1}\right) \mathrm{X}_{\mathrm{t}-1}+\mathrm{Z}_{\mathrm{t}}$

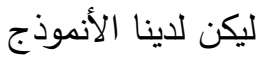

دورة النهاية بالدورة q (أن وجد) للأنموذج أعلاه تكون مستقرة مداريا(orbitally stable) اذا تحقق الثرط: $<1\left|\frac{\xi_{t+q}}{\xi_{t}}\right|$

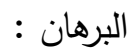

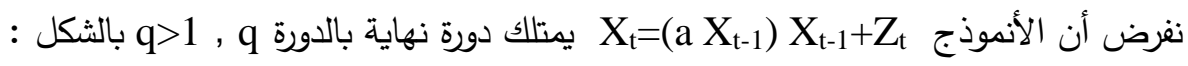
$\mathrm{X}_{\mathrm{t}}, \mathrm{X}_{\mathrm{t}+1}, \mathrm{X}_{\mathrm{t}+2}, \ldots, \mathrm{X}_{\mathrm{t}+\mathrm{q}}=\mathrm{X}_{\mathrm{t}}$

وهو مسار مغلق ومعزول .

; s=t ,t-1, $X_{S}+\xi_{s} \rightarrow$ على مسار قريب من دورة النهاية يمكن التعبير عنها X $\xi_{t}^{n} \rightarrow 0, \forall n \geq 2 \quad$ وبإلغاء تأثير نحصل على تصني

$X_{t}+\xi_{t}=a\left(X_{t-1}^{2}+2 X_{t-1} \xi_{t-1}+\xi_{t-1}^{2}\right)$

$\xi_{t}=\left(2 a X_{t-1}\right) \xi_{t-1}$

أي أن

$\xi_{t+q}=\left(2 a X_{t+q-1}\right) \xi_{t+q-1}$

t=t+q لتكن

ومنها نحصل على م

$\xi_{t+q}=\left(2 a X_{t+q-1}\right)\left(2 a X_{t+q-2}\right)\left(2 a X_{t+q-3}\right) \cdots\left(2 a X_{t}\right) \xi_{t}$

$\xi_{t+q}=\prod_{i=1}^{q}\left(2 a X_{t+q-i}\right) \xi_{t}$

أي أن دورة النهاية لأنموذج الانحدار الذاتي المتعدد الحدود (من الدرجة الثانية والمرتبة الاولى) يكون غير مراوح

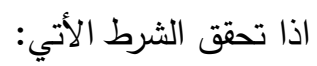
$\left|\prod_{i=1}^{q}\left(2 a X_{t+q-i}\right)\right|<1$

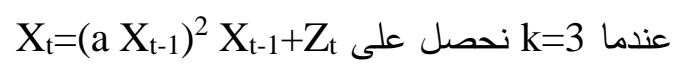

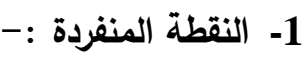

تكون النقاط المنفردة للأنموذج أعلاه هي النقطة الصفرية و

2-2-إستقراية النقطة المنفردة :-

باستخدام نفس الفرضيات السابقة وبتعويض هذه الفرضيات بالأنموذج أعلاه نحصل على

$\ldots(21) \xi_{t}=3 \xi_{t-1}$

المعادلة (21) تمثل أنموذج أنحدار ذاتي خطي من المرتبة الأولى وأن جذر المعادلة =3 أي أنه يقع خارج

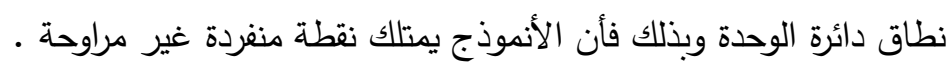

3- - إستقرارية دورة النهاية :-

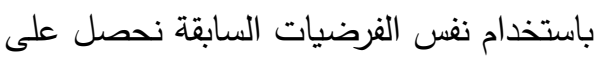

$X_{t}+\xi_{t}=a^{2}\left(X_{t-1}^{2}+2 X_{t-1} \xi_{t-1}+\xi_{t-1}^{2}\right)\left(X_{t-1}+\xi_{t-1}\right)$ 


$$
\xi_{t-1} \xi_{t}=\left(3 a^{2} X_{t-1}^{2}\right)
$$

$\xi_{t+q}=\prod_{i=1}^{q}\left(3 a^{2} X_{t+q-i}^{2}\right) \xi_{t}$

t=t+q لتكن

وبذلك فأن شرط إستقرارية دورة النهاية لأنموذج الانحدار الذاتي المتعدد الحدود (من الدرجة الثالثة والمرتبة

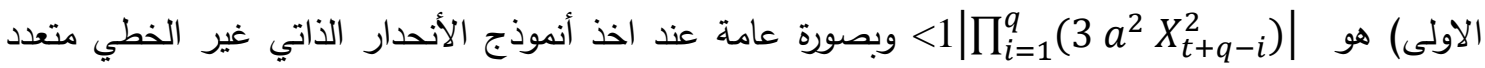
$\mathrm{X}_{\mathrm{t}}=(\mathrm{a} \mathrm{X} \mathrm{t}-1)^{\mathrm{k}}+\mathrm{Z}_{\mathrm{t}} \quad \mathrm{k}=1,2,3, \ldots$ الحدود ( من المرتبة الاولى والدرجة k) والذبي صيغته: 1- النقاط المنفردة لهذا الأنموذج هي النقطة الصفرية و النقاط 2- 2 إستقرارية النقاط المنفردة:

$$
\begin{aligned}
& \text { باستخدام الفرضيات التي تم تطبيقها على الانموذج عندما k=1,2 نحصل على } \\
& \text {,k=2,3,4,... } \quad \ldots(22) \xi_{t}=(k) \xi_{t-1}
\end{aligned}
$$

والذي تمثل أنموذج انحدار ذاتي خطي من المرتبة الأولى (وأن جذر المعادلة هو (k)) وبهذا فأن أنموذج الأنحدار الذاتي غير الخطي (متعدد الحدود من المرتبة الأولى والدرجة k) يمتلك نقاط منفردة غير صغرية الدانية

$$
\text { وغير مراوحة }
$$

\section{3- شرط إستقرارية دورة النهاية : وعراهه}

بالاعتماد على المبرهنة التي تم استخدامها في الحالات k=2,3 على الانموذج المعرف بالمعادلة (8)

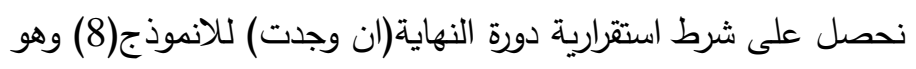

$<1$

$$
\text { ... (23) }\left|\prod_{i=1}^{q}\left((k) a^{k-1} X_{t+q-i}^{k-1}\right)\right|
$$

$$
\text { وبهذا يتم البرهان. }
$$

تم في هذا البحث دراسة الصفات الرياضية والإحصائية لأحد نماذج الانحدار الذاتي غير الخطي (انموذج متعدد الحدود من المرتبة الاولى والدرجة k ) ومن خلال الدراسة تبين ما يلي : 1- بعد إيجاد العزوم بطريقة (التعويض المتعاقب) تبين ان جميع العزوم المتمثلة (المعدل والتباين والتغاير) تعتمد على الزمن أي أن الأنموذج غير مراوح (من تعريف الاستمرارية ).

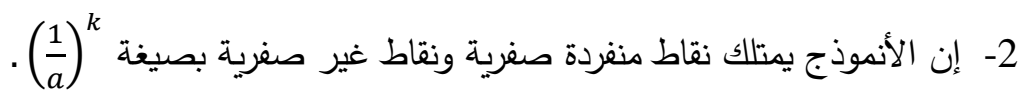

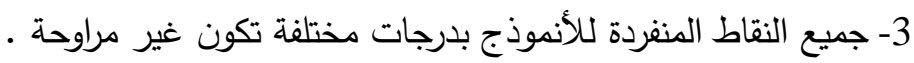
$<1\left|\prod_{\mathrm{i}=1}^{\mathrm{q}}\left((\mathrm{k}) \mathrm{a}^{\mathrm{k}-1} \mathrm{X}_{\mathrm{t}+\mathrm{q}-\mathrm{i}}^{\mathrm{k}-1}\right)\right|$

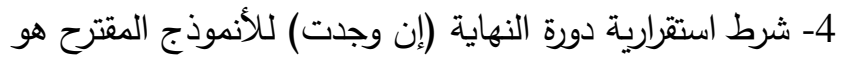
q > حيث 
|لمصادر

[1] البدراني , ظافر رمضان (2002) "دراسة في تشخيص نظم السيطرة التصادفية مع إثارة خاصة الى اسلوب

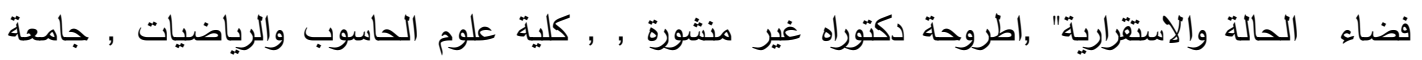

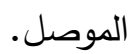

[2] السليم , إسراء سالم محمود السليم (2012) "دراسة استقرارية نماذج الانحدار الذاتي غير الخطية وبحدود دوال

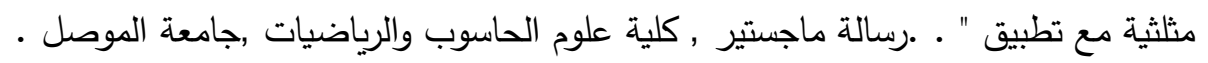

[3] خلف , حامد محمد خلف (2011) ,"دراسة استقرارية احد نماذج الانحدار الذاتي النسبي غير الخطي",بحث

منشور , كلية علوم الحاسوب والرياضيات , جامعة الموصل.

[4] فاندل, واللتر فاندل (1992) "السلاسل الزمنية من الوجهة التطبيقية ونماذج بوكس-كوكس" .

[5] نهال , نهال هاشم حميد (2009) "عكس نماذج الانحدار الذاتي المتقعطة الى مستمرة" .رسالة ماجستير , كلية

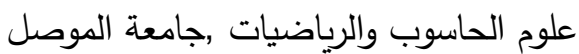

[6] Al-jalawy,Amed S.A., (2003), "Solution of difference equation using Z-transform technique" Msc. In applied mathematics unpuplished,Al-Nahrain university,Iraq.

[7] Chatifield , C. , (1978), "The Analysis of time series ", $4^{\text {th }}$ edition Chapman and Hall , London .

[8] Chen, S. ; S.A. Billings , (1989), "Modlling and analysis of non-linear time series", University of Southampton .

[9] Ozaki , T. , (1982), " Nonlinear Time series stochastic Processes and Dynamical system " Handbook of statistics, Vo1.5 , Itd .

[10] Ozaki , T. ,(1985), " Nonlinear Time series Models and Dynamical system " E.J. Hannan , P.R. Krishnaiah , M , M.Rao, eds. , Handbook of statistics, Vo1.5, PP.25-83 .

[11] Priestley ,M.B. , (1988), "Nonlinear And Nonstationary Time Series Analysis " London: ACADEMIC. Press .

[12] Witt , A. \& Kurths , J. \& Pikosvky , A. , (1998), " Testing statinoarity in time series ", Physical Review E 58:1800-1810 . 